\title{
SEXUALSTRAFTÄTER
}

\section{Gegen ältere Frauen besonders brutal}

— Frauen nach der Menopause erleiden bei einer Vergewaltigung deutlich schwerere Verletzungen als jüngere. In einer retrospektiven Studie verglich Louise Morgan vom Haven Camberwell King's College Hospital in London die Befunde von 122 Frauen nach der Menopause mit jenen von 130 jüngeren Frauen, die alle Opfer eines sexuellen Übergriffs geworden waren. Auffällig war nicht nur, dass ältere Opfer öfter genitale Verletzungen erlitten (37\% der vergewaltigten Frauen in der Postmenopause, verglichen mit $17 \%$ in der Prämenopause). $71 \%$ der vergewaltigten älteren Frauen erlitten zudem Läsionen an anderen Körperstellen, wobei sie signifikant häufiger ausgedehnte Hämatome aufwiesen. BJOG 2011;118:832-843.

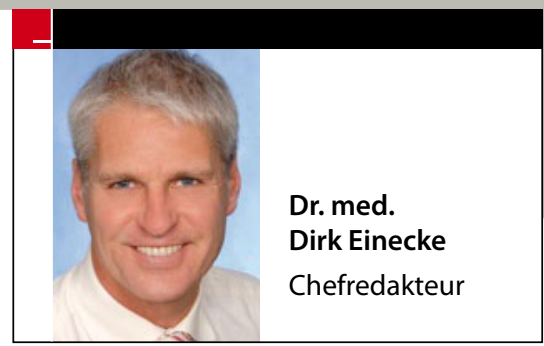

NEUER OUTCOME-PARAMETER

Lärm im Op-Saal -
Infektionsgefahr!

— Ob ein Patient nach einem chirurgischen Eingriff eine Infektion entwickelt, hängt auch vom Geräuschpegel während der Operation ab. Chirurgen der Universität Bern verfolgten offene Bauch-Operationen mit dem Schallpegelmessgerät. Von $35 \mathrm{~Pa}-$ tienten entwickelten sieben innerhalb von 30 Tagen eine Infektion an der Eingriffsstelle. Bei diesen Patienten hatte der mittlere Geräuschpegel während der Op. mit 43,5 Dezibel deutlich höher gelegen als bei den Patienten ohne eine solche Komplikation (25 Dezibel). „Ein erhöhter Geräuschpegel kann ein Indikator sein für ein chirurgisches Problem, eine belastende Umgebung oder mangelnde Disziplin", sagte Studienleiter Dr. Guido Beldi. „Jeder dieser Faktoren könnte das Risiko für postoperative Komplikationen erhöhen." Beldi wünscht sich deswegen weitere Studien, die sich mit den Ursachen für den „Radau“ im Op-Saal und seinem Einfluss auf die Leistung der Chirurgen befassen.

BJSurg 201;98:1021-25

\section{Mandeln und Blinddarm raus: Herzinfarktrisiko steigt}

- Wenn Mandeln oder Blinddarm vor dem 20. Lebensjahr entfernt werden, steigt das Risiko für einen Myokardinfarkt. In einer schwedischen Registerstudie ereigneten sich Herzinfarkte in den 23 Jahren nach einer Appendektomie um 33\% häufiger und nach einer Tonsillektomie um 44\% häufiger als ohne diese Eingriffe. Die absoluten Fall- zahlen waren allerdings gering, mit 47 Infarkten bei 27284 Personen ohne Mandeln und 89 Infarkten bei 54449 Personen ohne Wurmfortsatz. Da Tonsillen und Blinddarm sekundäre Lymphorgane sind, wird vermutet, dass sich die Entfernung ungünstig auf das Immunsystem auswirkt.

Eur Heart J 2011; Online-Publikation 31.Mai 2011

\section{www.SpringerMedizin.de}

\section{Laufend das Neueste über EHEC}

Bleiben Sie während der EHECEpidemie up to date! Das ständig aktualisierte EHEC-Dossier von springermedizin.de hilft Ihnen dabei: http://www.springermedizin.de/ehec 